PROCEEDINGS OF THE

AMERICAN MATHEMATICAL SOCIETY

Volume 139, Number 8, August 2011, Pages 2927-2931

S 0002-9939(2010)10707-4

Article electronically published on December 28, 2010

\title{
HARMONIC COHOMOLOGY OF SYMPLECTIC FIBER BUNDLES
}

\author{
OLIVER EBNER AND STEFAN HALLER
}

(Communicated by Jianguo Cao)

\begin{abstract}
We show that every de Rham cohomology class on the total space of a symplectic fiber bundle with closed Lefschetz fibers admits a Poisson harmonic representative in the sense of Brylinski. The proof is based on a new characterization of closed Lefschetz manifolds.
\end{abstract}

\section{INTRODUCTION AND MAIN RESULT}

Suppose $P$ is a Poisson manifold [10] with Poisson tensor $\pi$. Let $d$ denote the de Rham differential on $\Omega(P)$ and write $i_{\pi}$ for the contraction with the Poisson tensor. Recall that Koszul's [5] codifferential $\delta:=\left[i_{\pi}, d\right]=i_{\pi} d-d i_{\pi}$ satisfies $\delta^{2}=0$ and $[d, \delta]=d \delta+\delta d=0$. Differential forms $\alpha \in \Omega(P)$ with $d \alpha=0=\delta \alpha$ are called (Poisson) harmonic. Brylinski [2] asked for conditions on a Poisson manifold which imply that every de Rham cohomology class admits a harmonic representative.

In the symplectic case, this question has been settled by Mathieu. Recall that a symplectic manifold $(M, \omega)$ of dimension $2 n$ is called Lefschetz iff, for all $k$,

$$
[\omega]^{k} \wedge H^{n-k}(M ; \mathbb{R})=H^{n+k}(M ; \mathbb{R}) .
$$

According to Mathieu 6] (see [1] for an alternative proof) a symplectic manifold is Lefschetz iff it satisfies the Brylinski conjecture; i.e. every de Rham cohomology class of $M$ admits a harmonic representative.

In this paper we study the Brylinski problem for smooth symplectic fiber bundles 7. Recall that the total space of a symplectic fiber bundle $P \rightarrow B$ is canonically equipped with the structure of a Poisson manifold obtained from the symplectic form on each fiber. Locally, the Poisson structure on $P$ is product-like; that is, every point in $B$ admits an open neighborhood $U$ such that there exists a fiber preserving Poisson diffeomorphism $\left.P\right|_{U} \cong M \times U$. Here $M$ denotes the typical symplectic fiber, equipped with the corresponding Poisson structure, and $U$ is considered as a trivial Poisson manifold. This renders the symplectic foliation of $P$ particularly nice, for its leaves coincide with the connected components of the fibers of the bundle $P \rightarrow B$.

The aim of this paper is to establish the following result, providing a class of Poisson manifolds which satisfy the Brylinski conjecture.

Received by the editors April 13, 2010 and, in revised form, July 19, 2010.

2010 Mathematics Subject Classification. Primary 53D17.

Key words and phrases. Brylinski problem, Poisson manifolds, harmonic cohomology.

The first author was partially supported by the Austrian Science Fund, grant S9209.

The second author acknowledges the support of the Austrian Science Fund, grant P19392-N13.

(C)2010 American Mathematical Society 
Theorem 1. Let $M$ be a closed symplectic Lefschetz manifold, and suppose $P \rightarrow B$ is a smooth symplectic fiber bundle with typical symplectic fiber $M$. Then every de Rham cohomology class of $P$ admits a Poisson harmonic representative. Moreover, the analogous statement for compactly supported cohomology holds true.

This result, as well as a characterization of closed Lefschetz manifolds similar to Theorem 2 below, has been established in the first author's diploma thesis, employing sightly different methods than those of the present work; see [3].

The proof presented in Section 3 below is based on a handlebody decomposition $\emptyset=B_{0} \subseteq B_{1} \subseteq B_{2} \subseteq \cdots$ of $B$. Given a cohomology class of $P$, we will inductively produce representatives which are harmonic on $\left.P\right|_{B_{k}}$, for increasing $k$. The crucial problem, of course, is to extend harmonic forms across the handle, from $\left.P\right|_{B_{k}}$ to $\left.P\right|_{B_{k+1}}$. This issue is addressed in Theorem 2] see also Lemma 6 .

\section{EXTENSION OF HARMONIC FORMS}

Let $M$ be a closed symplectic manifold and consider the trivial symplectic fiber bundle $P:=M \times \mathbb{R}^{p} \times D^{q}$ where $D^{q}$ denotes the $q$-dimensional closed unit ball. In other words, the Poisson structure on $P$ is the product structure obtained from the symplectic form on $M$ and the trivial Poisson structure on $\mathbb{R}^{p} \times D^{q}$. Note that the boundary $\partial P=M \times \mathbb{R}^{p} \times \partial D^{q}$ is a Poisson submanifold. It turns out that the Lefschetz property of $M$ is equivalent to harmonic extendability of forms, from $\partial P$ to $P$.

To formulate this precisely, we need to introduce some notation that will be used throughout the rest of the paper. For every Poisson manifold $P$ we let $Z(P):=$ $\{\alpha \in \Omega(P) \mid d \alpha=0\}$ and $Z_{0}(P):=\{\alpha \in \Omega(P) \mid d \alpha=0=\delta \alpha\}$ denote the spaces of closed and harmonic differential forms, respectively. Moreover, we write $H_{0}(P):=\operatorname{ker}(d) \cap \operatorname{ker}(\delta) / \operatorname{img}(d) \cap \operatorname{ker}(\delta)$ for the space of de Rham cohomology classes which admit a harmonic representative, $H_{0}(P) \subseteq H(P)$. If $\iota: S \hookrightarrow P$ is a Poisson submanifold, then the relative complex $\Omega(P, S):=\left\{\alpha \in \Omega(P) \mid \iota^{*} \alpha=0\right\}$ is invariant under $\delta$, and we define the relative harmonic cohomology $H_{0}(P, S) \subseteq$ $H(P, S)$ in an analogous manner. Finally, if $Q$ is a Poisson manifold and $B$ is a smooth manifold, we let $\Omega_{\mathrm{vc}}(Q \times B)$ denote the space of forms with vertically compact support (with respect to the projection $Q \times B \rightarrow Q$ ), and define the harmonic cohomology with vertically compact supports $H_{\mathrm{vc}, 0}(Q \times B) \subseteq H_{\mathrm{vc}}(Q \times B)$ in the obvious way.

Here is the main result that will be established in this section.

Theorem 2. Let $M$ be a closed symplectic manifold, suppose $p, q \in \mathbb{N}_{0}$, and consider the Poisson manifold $P:=M \times \mathbb{R}^{p} \times D^{q}$. Then the following are equivalent:

(i) $M$ is Lefschetz; i.e. $H_{0}(M)=H(M)$ according to [6].

(ii) $H_{0}(P, \partial P)=H(P, \partial P)$.

(iii) $H_{\mathrm{vc}, 0}(P \backslash \partial P)=H_{\mathrm{vc}}(P \backslash \partial P)$ with respect to the projection along $D^{q} \backslash \partial D^{q}$.

(iv) If $\alpha \in Z(P)$ is harmonic on a neighborhood of $\partial P$, then there exists $\beta \in \Omega(P)$, supported on $P \backslash \partial P$, so that $\alpha+d \beta$ is harmonic on $P$.

(v) If $\alpha \in Z(P)$ and $\delta \iota^{*} \alpha=0$, then there exists $\beta \in \Omega(P)$ with $\iota^{*} \beta=0$, so that $\alpha+d \beta$ is harmonic on $P$. Here $\iota: \partial P \hookrightarrow P$ denotes the canonical inclusion.

An essential ingredient for the proof of Theorem 2 is the following $d \delta$-lemma.

Lemma 1 ( $d \delta$-Lemma, 4, 8]). A closed symplectic manifold is Lefschetz if and only if $\operatorname{ker}(\delta) \cap \operatorname{img}(d)=\operatorname{img}(d \delta)$. 
We will also make use of the following averaging argument.

Lemma 2. Suppose $G$ is a connected compact Lie group acting on a Poisson manifold $P$ via Poisson diffeomorphisms, and let $r: \Omega(P \times I) \rightarrow \Omega(P \times I)^{G}$, $r(\alpha):=\int_{G} g^{*} \alpha d g$, denote the standard projection onto the space of $G$-invariant forms, $I:=[0,1]$. Then there exists an operator $A: \Omega(P \times I) \rightarrow \Omega(P \times I)$, commuting with $d, i_{\pi}$ and $\delta$, so that $A(\alpha)=\alpha$ in a neighborhood of $P \times\{1\}$ and $A(\alpha)=r(\alpha)$ in a neighborhood of $P \times\{0\}$, for all $\alpha \in \Omega(P \times I)$.

Proof. Choose finitely many smoothly embedded closed balls $D_{i} \subseteq G$ such that $\bigcup_{i} \stackrel{\circ}{D}_{i}=G$. Let $\lambda_{i}$ denote a partition of unity on $G$ so that $\operatorname{supp}\left(\lambda_{i}\right) \subseteq D_{i}$. Choose smooth contractions $h_{i}: D_{i} \times I \rightarrow G$ so that $h_{i}(g, t)=g$ for $t \leq 1 / 3$ and $h_{i}(g, t)=e$ for $t \geq 2 / 3, g \in D_{i}$. Here $e$ denotes the neutral element of $G$. Using the maps

$$
\phi_{i, g}: P \times I \rightarrow P \times I, \quad \phi_{i, g}(x, t):=\left(h_{i}(g, t) \cdot x, t\right), \quad g \in D_{i},
$$

we define the operator $A: \Omega(P \times I) \rightarrow \Omega(P \times I)$ by

$$
A(\alpha):=\sum_{i} \int_{D_{i}} \lambda_{i}(g) \phi_{i, g}^{*} \alpha d g,
$$

where integration is with respect to the invariant Haar measure of $G$. It is straightforward to verify that $A$ has the desired properties; the relations $\left[A, i_{\pi}\right]=0=[A, \delta]$ follow from the fact that each $\phi_{i, g}$ is a Poisson map.

The following application of Lemma 2 will be used in the proof of Theorem 2

Lemma 3. Let $M$ be a symplectic manifold and consider the Poisson manifold $P:=M \times \mathbb{R}^{p} \times A^{q}$ where $A^{q}:=\left\{\xi \in \mathbb{R}^{q}\left|\frac{1}{2} \leq\right| \xi \mid \leq 1\right\}$ denotes the $q$-dimensional annulus. Moreover, suppose $\alpha \in \Omega(P)$ is harmonic on a neighborhood of $\partial_{+} P:=$ $M \times \mathbb{R}^{p} \times \partial D^{q}$. Then there exist $\beta \in \Omega(P)$, supported on $P \backslash \partial_{+} P$, and $\beta_{1}, \beta_{2} \in$ $Z_{0}(M)$, so that $\tilde{\alpha}:=\alpha+d \beta$ is harmonic on $P$ and $\tilde{\alpha}=\sigma^{*} \beta_{1}+\sigma^{*} \beta_{2} \wedge \rho^{*} \theta$ in a neighborhood of $\partial_{-} P:=M \times \mathbb{R}^{p} \times \frac{1}{2} \partial D^{q}$. Here $\sigma: P \rightarrow M$ and $\rho: P \rightarrow \partial D^{q}$ denote the canonical projections, and $\theta$ denotes the standard volume form on $\partial D^{q} 1$

Proof. W.l.o.g. we may assume $\alpha \in Z_{0}(P)$ and $\alpha=\tau^{*} \gamma$ in a neighborhood of $\partial_{-} P$ where $\gamma \in Z_{0}\left(M \times \partial D^{q}\right)$ and $\tau=(\sigma, \rho): P \rightarrow M \times \partial D^{q}$ denotes the canonical projection. Applying the operator $A$ from Lemma 2 to $\alpha$, we obtain $\tilde{\alpha} \in Z_{0}(P)$ so that $\tilde{\alpha}=\alpha$ in a neighborhood of $\partial_{+} P$ and $\tilde{\alpha}=\tau^{*} \tilde{\gamma}$ in a neighborhood of $\partial_{-} P$, where $\tilde{\gamma} \in Z_{0}\left(M \times \partial D^{q}\right)$ is $S O(q)$-invariant. We conclude that $\tilde{\gamma}$ is of the form $\tilde{\gamma}=\beta_{1}+\beta_{2} \wedge \theta$ with $\beta_{1}, \beta_{2} \in Z_{0}(M)$, whence $\tilde{\alpha}=\sigma^{*} \beta_{1}+\sigma^{*} \beta_{2} \wedge \rho^{*} \theta$ in a neighborhood of $\partial_{-} P$. Clearly, there exists $\beta \in \Omega(P)$, supported on $P \backslash \partial_{+} P$, such that $\tilde{\alpha}-\alpha=d \beta$.

Lemma 4. Let $P$ be a Poisson manifold, and suppose $B$ is an oriented smooth manifold with boundary. Then integration along the fibers $\int_{B}: \Omega_{\mathrm{vc}}(P \times B) \rightarrow \Omega(P)$ commutes with $i_{\pi}$ and $\delta$.

Proof. The relation $i_{\pi} \int_{B} \alpha=\int_{B} i_{\pi} \alpha$ is obvious. Combining this with Stokes' theorem, that is $\left[\int_{B}, d\right]=\int_{\partial B} \iota^{*}$, we obtain

$$
\left[\int_{B}, \delta\right]=\left[\int_{B},\left[i_{\pi}, d\right]\right]=\left[\left[\int_{B}, i_{\pi}\right], d\right]+\left[i_{\pi},\left[\int_{B}, d\right]\right]=\left[i_{\pi}, \int_{\partial B} \iota^{*}\right]=0 .
$$

Here $\iota: P \times \partial B \hookrightarrow P \times B$ denotes the canonical inclusion.

\footnotetext{
${ }^{1}$ To be specific, in the case $q=1$ we assume $\theta(-1)=-1 / 2$ and $\theta(1)=1 / 2$, so that $\int_{\partial D^{q}} \theta=1$ with respect to orientation on $\partial D^{q}$ induced from the standard orientation of $D^{q}$.
} 
Lemma 5. Suppose $Q$ is a Poisson manifold, and consider the Poisson manifold $P:=Q \times D^{q}$. Then the Thom (Künneth) isomorphism restricts to an isomorphism of harmonic cohomology; i.e. $H_{0}^{*-q}(Q)=H_{\mathrm{vc}, 0}^{*}(P \backslash \partial P)=H_{0}^{*}(P, \partial P)$.

Proof. Choose $\eta \in \Omega^{q}\left(D^{q}\right)$, supported on $D^{q} \backslash \partial D^{q}$, such that $\int_{D^{q}} \eta=1$. Clearly, the chain map $\Omega(Q) \rightarrow \Omega_{\mathrm{vc}}(P \backslash \partial P) \subseteq \Omega(P, \partial P), \alpha \mapsto \alpha \wedge \eta$, commutes with $\delta$. This map induces the Thom isomorphism which therefore preserves harmonicity. Its inverse is induced by integration along the fibers $\int_{D^{q}}: \Omega(P, \partial P) \rightarrow \Omega(Q)$, and this commutes with $\delta$ too; see Lemma 4 .

Now the table is served, and we proceed to the

Proof of Theorem 2. Set $Q:=M \times \mathbb{R}^{p}$ and note that the isomorphism $H(Q)=$ $H(M)$ induced by the canonical projection restricts to an isomorphism of harmonic cohomology $H_{0}(Q)=H_{0}(M)$. The equivalence of the first three statements thus follows from Lemma 5. Let us continue by showing that (iii) implies (iv). Assume $\alpha \in Z(P)$ is harmonic on a neighborhood of $\partial P$. Let $\rho: P \backslash\left(M \times \mathbb{R}^{p} \times\{0\}\right) \rightarrow \partial D^{q}$ and $\sigma: P \rightarrow M$ denote the canonical projections. In view of Lemma 3 , we may w.l.o.g. assume $\alpha=\sigma^{*} \beta_{1}+\sigma^{*} \beta_{2} \wedge \rho^{*} \theta$ in a neighborhood of $\partial P$ where $\beta_{1}, \beta_{2} \in$ $Z_{0}(M)$ and $\theta$ denotes the standard volume form on $\partial D^{q}$. Using Stokes' theorem for integration along the fiber of $M \times D^{q} \rightarrow M$, we obtain

$$
\beta_{2}=\int_{\partial D^{q}} j^{*} \alpha=-d \int_{D^{q}} j^{*} \alpha \in \operatorname{img}(d) \cap \operatorname{ker}(\delta),
$$

where $j: M \times D^{q} \rightarrow M \times\{0\} \times D^{q} \subseteq P$ denotes the canonical inclusion. By the $d \delta$ lemma, Lemma 1 we thus have $\beta_{2}=d \delta \gamma$ for some differential form $\gamma$ on $M$. Let $\lambda$ be a smooth function on $P$, identically 1 in a neighborhood of $\partial P$, identically 0 near $M \times \mathbb{R}^{p} \times\{0\}$, and constant in the $M$-direction. Then $\tilde{\alpha}:=\sigma^{*} \beta_{1}+d\left(\delta \sigma^{*} \gamma \wedge \lambda \rho^{*} \theta\right)$ is harmonic on $P$, and $\alpha-\tilde{\alpha}=0$ in a neighborhood of $\partial P$. Hence, using (iii), we find $\beta \in \Omega(P)$, supported on $P \backslash \partial P$, so that $\alpha-\tilde{\alpha}+d \beta$ is harmonic on $P$. Thus, $\beta$ has the desired property. Let us next show that (iv) implies (四). Suppose $\alpha \in Z(P)$ and $\delta \iota^{*} \alpha=0$. Clearly, there exists $\beta_{1} \in \Omega(P)$, with $\iota^{*} \beta_{1}=0$, so that $\tilde{\alpha}:=\alpha+d \beta_{1}$ satisfies $r^{*} \tilde{\alpha}=\tilde{\alpha}$ near $\partial P$, where $r: P \backslash\left(M \times \mathbb{R}^{p} \times\{0\}\right) \rightarrow \partial P$ denotes the canonical radial retraction. Particularly, $\tilde{\alpha}$ is harmonic on a neighborhood of $\partial P$. According to (iv) there exists $\beta_{2} \in \Omega(P)$, supported on $P \backslash \partial P$, so that $\tilde{\alpha}+d \beta_{2}$ is harmonic on $P$. The form $\beta:=\beta_{1}+\beta_{2}$ thus has the desired property. Obviously, ( $(\mathbb{\nabla})$ implies (iii).

\section{Proof of Theorem 1}

Choose a proper Morse function $f$ on $B$, bounded from below, so that the preimage of each critical value consists of a single critical point [9. We label the critical values in increasing order $c_{0}<c_{1}<\cdots$, and choose regular values $r_{k}$ so that $c_{k-1}<r_{k}<c_{k}$. By construction, the sublevel sets $B_{k}:=\left\{f(x) \leq r_{k}\right\}$ provide an increasing filtration of $B$ by compact submanifolds with boundary, $\emptyset=B_{0} \subseteq B_{1} \subseteq B_{2} \subseteq \cdots$. The statement in Theorem 1 is an immediate consequence of the following:

Lemma 6. Suppose $\alpha \in Z(P)$ is a closed form which is harmonic on a neighborhood of $\left.P\right|_{B_{k}}$. Then there exists $\beta \in \Omega(P)$, supported on $\left.P\right|_{B_{k+2} \backslash B_{k}}$, such that $\alpha+d \beta$ is harmonic on a neighborhood of $\left.P\right|_{B_{k+1}}$. 
Proof. Let $q$ denote the Morse index of the unique critical point in $B_{k+1} \backslash B_{k}$, and set $p:=\operatorname{dim} B-q$. Recall [9] that there exists an embedding $j: \mathbb{R}^{p} \times D^{q} \rightarrow B_{k+1} \backslash \stackrel{\circ}{B}_{k}$ so that $j\left(\mathbb{R}^{p} \times \partial D^{q}\right)=j\left(\mathbb{R}^{p} \times D^{q}\right) \cap \partial B_{k}$. Moreover, there exists a vector field $X$ on $B$, supported on $B_{k+2} \backslash B_{k}$, so that its flow $\varphi_{t}$ maps $B_{k+1}$ into any given neighborhood of $\partial B_{k} \cup j\left(\{0\} \times D^{q}\right)$, for sufficiently large $t$.

Trivializing the symplectic bundle $P$ over the image of $j$, we obtain an isomorphism of Poisson manifolds $j^{*} P \cong M \times \mathbb{R}^{p} \times D^{q}$. Using Theorem 2(iv), we may thus assume that there exists an open neighborhood $U$ of $\partial B_{k} \cup j\left(\{0\} \times D^{q}\right)$ so that $\alpha$ is harmonic on $\left.P\right|_{U}$. Let $\tilde{X}$ denote the horizontal lift of $X$ with respect to a symplectic connection [7 on $P$, and denote its flow at time $t$ by $\tilde{\varphi}_{t}$. Clearly, each $\tilde{\varphi}_{t}$ is a Poisson map. Moreover, there exists $t_{0}$ so that $\tilde{\varphi}_{t_{0}}$ maps $\left.P\right|_{B_{k+1}}$ into $\left.P\right|_{U}$. Thus, $\tilde{\varphi}_{t_{0}}^{*} \alpha$ is harmonic on $\left.P\right|_{B_{k+1}}$. Furthermore, $\tilde{\varphi}_{t_{0}}^{*} \alpha-\alpha=d \beta$, where $\beta:=\int_{0}^{t_{0}} \tilde{\varphi}_{t}^{*} i_{\tilde{X}} \alpha d t$ is supported on $\left.P\right|_{B_{k+2} \backslash B_{k}}$.

\section{REFERENCES}

[1] R. Bott and L.W. Tu, Differential forms in algebraic topology. Graduate Texts in Mathematics 82, Springer-Verlag, New York-Berlin, 1982. MR658304 (83i:57016)

[2] J.-L. Brylinski, A differential complex for Poisson manifolds, J. Differential Geom. 28(1988), 93-114. MR950556 (89m:58006)

[3] O. Ebner, Harmonic cohomology on Poisson manifolds, diploma thesis, University of Graz, 2009.

[4] V. Guillemin, Symplectic Hodge theory and the d $\delta$-lemma, preprint, MIT, 2001.

[5] J.-L. Koszul, Crochet de Schouten-Nijenhuis et cohomologie, in The mathematical heritage of Élie Cartan. Astérisque 1985, 257-271. MR837203 (88m:17013)

[6] O. Mathieu, Harmonic cohomology classes of symplectic manifolds, Comment. Math. Helv. 70(1995), 1-9. MR.1314938(96e:58004)

[7] D. McDuff and D. Salamon, Introduction to symplectic topology. Oxford University Press, New York, 1998. MR1698616 (2000g:53098)

[8] S.A. Merkulov, Formality of canonical symplectic complexes and Frobenius manifolds, Internat. Math. Res. Notices 14(1998), 727-733. MR.1637093 (99j:58078)

[9] J. Milnor, Morse theory. Annals of Mathematics Studies 51, Princeton University Press, Princeton, N.J., 1963. MR.0163331 (29:634)

[10] I. Vaisman, Lectures on the geometry of Poisson manifolds. Progress in Mathematics 118, Birkhäuser Verlag, Basel, 1994. MR1269545 (95h:58057)

[11] D. Yan, Hodge structure on symplectic manifolds, Adv. Math. 120(1996), 143-154. MR $1392276(97 \mathrm{e}: 58004)$

Institute of Geometry, Technische Universität Graz, Kopernikusgasse 24/IV, A-8010 Graz, Austria

E-mail address: o.ebner@tugraz.at

Department of Mathematics, University of Vienna, Nordbergstrasse 15, A-1090, Vienna, Austria

E-mail address: stefan.haller@univie.ac.at 